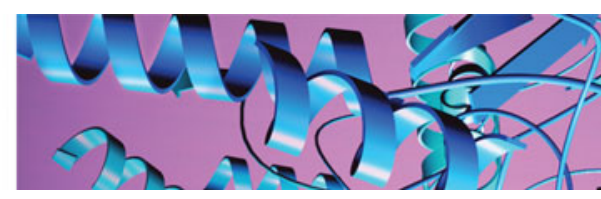

\title{
PERSPECTIVE
}

\section{A twisting story: how a single gene twists a snail? Mechanogenetics}

\author{
Reiko Kuroda* \\ Institute for Science and Technology, Tokyo University of Science, Noda, Chiba 278-8510, Japan \\ Quarterly Reviews of Biophysics (2015), 48(4), pages 445-452 doi:10.1017/S0033583515000098
}

Abstract. Left-right (1-r) symmetry breaking and the establishment of asymmetric animal body plan during embryonic development are fundamental questions in nature. The molecular basis of l-r symmetry breaking of snails is a fascinating topic as it is determined by a maternal single handedness-determining locus at a very early developmental stage. This perspective describes the current state of the art of the chiromorphogenesis, mainly based on our own work, i.e. the first step of $1-r$ symmetry breaking, as proven by our "Mechanogenetics", before the start of zygotic gene expression, transfer of chirality information to the cell-fate determining stage, and the expression of nodal at the blastula stage. The Nodal signalling pathway is a common mechanism in vertebrates' chiromorphogenesis in later development. Studies on snails, especially Lymnaea (L.) stagnalis, shall give important insights into the molecular basis of chiromorphogenesis not only in Lophotrochozoa but in vertebrates as well.

Key words: Chirality chiromorphology, left-right symmetry, breaking developmental biology, snail handedness.

\section{Introduction}

Chirality is expressed throughout nature, whether microscopic or macroscopic, and whether animate or inanimate. Some molecules are either chiral or non-chiral, and billions of them self-assemble to form either chiral or non-chiral crystals by way of chiral discrimination at the molecular level. In the biological world, chirality is also observed at all levels of hierarchy, from the macroscopic individual organisms down to molecules. At the molecular level, biological world is totally homochiral; i.e. all living organisms on Earth use molecules of a unique invariant handedness: only D-(deoxy) ribose in nucleic acids and only L-amino acids in proteins. At the macroscopic level, most animals appear symmetric externally, but exhibit chirality within the body cavity, i.e. in terms of asymmetric organ position, directional organ looping and lateralized organ function in the larva and adult. Thus, both in animate and inanimate domains, chirality is an excellent approach for studying the link between macroscopic and microscopic phenomena.

\footnotetext{
* Authors for correspondence: R. Kuroda, Research Institute for Science and Technology, Tokyo University of Science, Noda, Chiba 278-8510, Japan. Tel: +81-4-7124-1501; Email: rkuroda@rs.tus.acp
}

I have been interested in the molecular basis of left-right (l-r) symmetry breaking of animal body plan that starts from a single fertilized egg, as it is determined genetically by intricate molecular interactions among genes and proteins. The directions of anterior-posterior and dorsal-ventral axes during the embryonic morphogenesis are determined primarily, and they have been well characterized. In contrast, the $1-r$ direction emerges secondarily to the other two axes, and unlike the two axes which define dichotomies in biological and physical attributes such as head and tail, and dorsal and ventral, no functional difference is inherent to the left and right distinction. Embryonic morphogenesis along the $1-r$ axis has only recently begun to be elucidated. Several mechanisms for $1-r$ asymmetry determination have been proposed for vertebrates and invertebrates. In vertebrates, there is a general agreement that $1-r$ asymmetry is determined by directional nodal flow across the embryonic midline at a late development stage. Several unified models have been proposed, however, still little is known about the initial step of handedness determination for both in vertebrates and invertebrates (see recent Review articles by Blum et al. 2014; Coutelis et al. 2014; Vandenberg \& Levin, 2013, and references therein). 
I conceived the study on snails thinking that snails may become an ideal model system to study the initial step of chirality determination. There are several unique features in snails. First, the chirality is determined by maternal genetic inheritance. Namely, it is not the genotype of the individual but that of the mother that determines the chirality. The maternal inheritance is a crucial advantage as the chirality is determined at the early developmental stage. Recently, we proved that chirality is determined by a single locus (Hosoiri et al. 2003; Kuroda, 2014; Kuroda et al. 2009; Shibazaki et al. 2004), although it had been postulated based on the early 20th century work (Boycott et al. 1930; Sturtevant, 1923). Secondly, snails are hermaphrodite, reproducing offspring by self- and cross-fertilization, which is advantageous in tracing genetic traits. Thirdly, the genetically determined chirality depends on species, ca. 90\% dextral only, $10 \%$ sinistral only, and rare dimorphic with both chirality within a species. Fourthly, most snails display chirality not only internally but externally as well at the macroscopic level, which makes experiments easier.

We embarked on chiromorphology study using dimorphic fresh water snail, Lymnaea stagnalis. As the species had not been well studied compared with Lymnaea peregra (Freeman \& Lundelius, 1982) except for the detailed observation of development (Meshcheryakov, 1990), we studied the breeding behaviour (Hosoiri et al. 2003), correlation of cleavage pattern and organismal morphology (Shibazaki et al. 2004), cytoplasm injections to the 1-cell embryos of opposite chirality (Kuroda, 2014), cytoskeletal dynamics during spiral cleavages (Shibazaki et al. 2004), expression of nodal-Pitx genes (Kuroda et al. 2009), creation of mirrorimage healthy animals by twisting blastomeres at the third cleavage (Kuroda et al. 2009), etc. We also constructed backcrossed congenic animals (Hosoiri et al. 2003; Kuroda, 2014; Kuroda et al. 2009; Shibazaki et al. 2004) and carried out positional cloning in order to identify the handedness determining gene(s).

\section{Cytoskeletal dynamics during the third cleavage}

Non-mirror image relationship

The Spiralia (Lophotrochozoa) is one of the three major clades of bilaterian metazoan which includes molluscs and annelids. The Spiralian clade of animals are morphologically diverse but exhibit canonical spiral cleavage. Spiral cleavage where the cleavage planes are at oblique angles to the animal-vegetal $(\mathrm{A}-\mathrm{V})$ axis of the egg is characterized by the alternating clockwise and anticlockwise rotation particularly during the 3rd-5th cleavages (Verdonk \& van der Biggelaar, 1983). The correlation of the shell-coiling direction with the handedness of the third blastomere cleavage (i.e. from the four-cell to the eight-cell stage) has been known since 1894 (Crampton, 1894). We closely analysed the temporal and spatial behaviour of cytoskeletons of the dextral and the sinistral L. stagnalis during the early cleavages by staining filamentous actin with fluorescently labelled phalloidin and by visualizing spindles with indirect immunofluorescence with anti- $\beta$-tubulin antibody.

Contrary to what had been believed and illustrated in many standard textbooks, we revealed non-mirror-image cytoskeletal dynamics for the sinistral and dextral embryos at the most critical chiromorphogenetic step (Shibazaki et al. 2004). A helical deformation of blastomere shape was observed for the dextral embryos at the animal hemisphere in metaphase to anaphase (Fig. $1 a \mathrm{I}$ ), as described 'spiral deformation (SD)' (Meshcheryakov, 1978). Consequently, a quartet of micromeres protruded and budded off from the sister macromeres dextrotropically and rotated in a clockwise sense when viewed from the animal pole. In metaphase-anaphase, spindles were oriented with helicity along the A-V axis. We named this spindle inclination (SI) (Fig. 1a I). In marked contrast, neither SD nor SI was observed in metaphase to anaphase of sinistral embryos and each blastomere presented a symmetrical shape. Thus, the new micromeres emerged almost directly above the paired macromeres along the $\mathrm{A}-\mathrm{V}$ axis. Chirality was not obvious until furrow ingression, when new micromeres rotated levotropically. The spindles in metaphase-anaphase did not exhibit 'reversed' helicity but were positioned radially, devoid of chirality, and almost parallel to the $\mathrm{A}-\mathrm{V}$ axis (Fig. $1 a$ II). The blastomere shape and the spindle orientation as well as the timing of chiromorphogenesis are different for the dextral and sinistral embryos within a species, and are far from enantiomorphs (Shibazaki et al. 2004).

\section{$S D$ depends on actin polymerization}

When the dextral embryos were treated with actin depolymerization agents, latrunculin $\mathrm{A}$ or $\mathrm{B}$, they showed a concomitant absence of SD and SI (Fig. $1 b$ I left), resembling sinistral embryos (Fig. $1 a$ II). The treatment of sinistral embryos showed no change (Fig. $1 b$ II left). When the dextral embryos in prophase were treated with $200 \mathrm{nM}$ nocodazole, microtubule depolymerization agent, they did not form spindles as expected, but exhibited SD slightly more prominent (Fig. $1 b$ I right) than that of untreated embryos (Fig. $1 a \mathrm{I}$ ). Nocodazole treated sinistral embryos are devoid of spindles and the symmetrical shape of the blastomeres remained (Fig. $1 b$ II right). These results suggest that actin polymerization is involved in the dextral-specific SD. The cause and effect of spindle orientation and blastomere shape has become clear: spindles do not deform blastomeres but the blastomere deformation orients spindles. It is interesting to note that there seem to be some cross-talk between the actins and microtubules as the extent of SD enhances when the formation of spindles is inhibited (Shibazaki et al. 2004). 
(a)

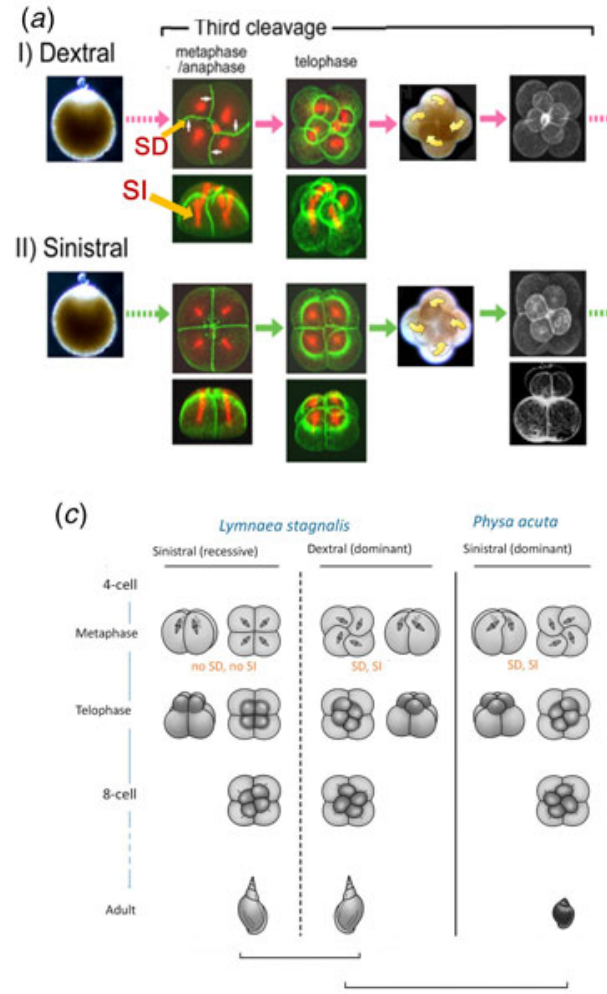

(b)

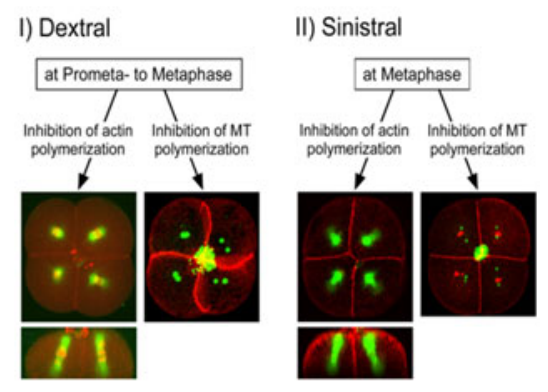

Fig. 1. The third cell cleavage of L. stagnalis for the dextral (I) and sinistral (II) embryos, and the schematic representation including P. acuta. (a) SD and SI occur only in the dextral embryo. Filamentous actin: green; microtubules: red. (b) Dextral-specific SD and SI are inhibited by treatment with an actin depolymerizing agent. F-actin and DNA: red; microtubules: green. (c) Schematic representation of non-mirror image relationship of the mirror-image snails within a species but mirror image relationship across species.

\section{Levotropical SD and SI of Physa (P.) acuta}

We found that P. acuta, a monomorphic sinistral gastropod, exhibits substantial SD and SI levotropically (Fig. 1c). Nocodazole and latrunculin A treatments on $P$. acuta embryos induced similar results to those observed for the dextral L. stagnalis, i.e. SD without formation of spindles for the nocodazole treatment, and loss of SD and SI for latrunculin A treatment. Although the recessive sinistral embryos of dextral-dominant L. stagnalis and $L$. peregra do not exhibit a mirror-image cleavage pattern of their respective dextral embryos at the third cleavage, cleavage pattern of the embryos of sinistral only P. acuta displays a mirror image pattern of dominant dextral Lymnaes. Figures in standard textbooks illustrate a mirror-image relationship during the embryogenesis for the enantiomorphic pair of snails within a species; however, they must be amended to the enantiomorphic pair across species as in Fig. $1 c$.

\section{Functions of SD and SI}

SD and SI are unique features of dominantly handed snails at the eight-cell stage. We have observed that only half of the embryos grew to normal snails in the case of recessive sinistral L. stagnalis, which is in sharp contrast to more than $90 \%$ in the dominant dextral embryos. This is due to the frequent incident of insufficient and heterogeneous rotation of micromeres at the third cleavage of the sinistral embryos, resulting in death before hatching. This clearly shows the role of SD to assure correct and sufficient rotation of blastomeres and the tilting of spindles, and hence the correct micromere-macromere contacts at the eight-cell stage (Kuroda, 2014).

\section{The body handedness is determined by a single locus that control cytoskeletal dynamics}

To prove that the handedness is determined by a single gene or genes at closely linked loci, we carried out backcross breeding between inbred dextral and sinistral strains of L. stagnalis cultivated in our laboratory. We used dextral animals as donor 'fathers' and sinistral animals as recurrent 'mothers', and the chirality-determining part of the genome from dextral inbred strain is introgressed on the genetic background of the sinistral inbred strain. Each of the backcross progeny obtained was typed for the chirality of the next generation oviposited by it, because the genotype for the handedness locus emerges as filial chirality. We have carried out serial backcrossing experiments (Kuroda et al. 
2009). We eventually succeeded in constructing the congenic strain $\mathrm{F}_{10}$ that on average inherit $99.9 \%$ of the sinistral-derived genome and only $0 \cdot 1 \%$ of the dextral strainderived genome. All the dextral embryos oviposited by $\mathrm{F}_{10}$ animals that inherited the dextrality gene(s) within the $0 \cdot 1 \%$ of the dextral-derived genome exhibited dextrotropical rotation with $\mathrm{SD} / \mathrm{SI}$ at the third cleavage, and were grown to possess dextral body shape without an exception. Equally, all the sinistral embryos oviposited by $F_{10}$ animals that do not inherit the dextrality-determining gene(s) showed levotropical rotation without SD/SI, and were grown to possess sinistral body shape, again without an exception (Kuroda, 2014; Kuroda et al. 2009). This is a strong indication that dextrality is determined by a single gene or by genes at closely linked loci. This was recently confirmed by DNA mapping using RAD-Seq and Fibre-FISH techniques (Liu et al. 2013). Furthermore, we could show that the gene dictates the cytoskeletal dynamics at the third cleavage.

\section{Creation of mirror-image animals by twisting blastomeres at the third cleavage}

\section{Cause and effect}

To prove directly the role of blastomere arrangements on the organismal handedness, we carried out "Mechanogenetics" and reversed the genetically specified third-cleavage directions by micromanipulation both in sinistral and dextral embryos of L. stagnalis. At metaphase-anaphase (for dextral snails) or telophase (for sinistral snails) of the third cleavage when embryonic chirality is about to be established, we pushed, by thin glass rods, the animal surface of each blastomere gently in the directions opposite to the normal third cleavage until contacts between newly formed adjacent pairs of a micromere and a macromere were established. We incubated the manipulated embryos in a glass capillary tube, and after about 17 days $1 / 3$ to $1 / 2$ of the inverted embryos developed into juvenile snails, and then to adults. Remarkably, all of them are situs inversus, and neither situs solitus nor situs ambiguous were observed (Fig. 2). We could show that the effect of the blastomere chirality at the third cleavage continues throughout the whole subsequent developmental programme (Kuroda et al. 2009).

\section{Twisting of embryos of P. acuta, a sinistral-only gastropod}

One may argue that the success in reversing genetically determined handedness by mechanical manipulation is due to the fact that L. stagnalis is dimorphic, which may somehow have a potential to accommodate the reversing physical force. By similar twisting of the embryos at the third cleavage, we obtained situs inversus for sinistral-only $P$. acuta as well (Abe et al. 2014). Thus, the creation of mirror-image individuals is not restricted to dimorphic species. Similar handedness-determining mechanisms must operate for L. stagnalis and P. acuta.

\section{Eight-cell stage is the key determinant step}

By similar mechanical manipulation, we altered the directions of blastomere rotations of both the sinistral and dextral embryos at the second cleavage to produce reversed blastomere configuration at the four-cell stage. However, the manipulated embryos all reverted to the original-type configuration at the third cleavage (Kuroda et al. 2009). We also observed that sinistral embryos occasionally showed dextral-type blastomere arrangement at the four-cell stage even inside the egg capsules, but they showed normal counterclockwise cleavage at the third division (Kuroda et al. 2009). It was reported that chirality is distinguishable as early as the second or even the first cleavage (Meshcheryakov \& Beloussov, 1975); however, our micromanipulation experiments have proven that the macromere-micromere cell contacts at the eight-cell stage embryo is the first determining step for asymmetric development.

\section{Not epigenetics}

The reversed-coiled snails were fertile, and produced sinistral or dextral progenies dictated by their genotype and not by the reversed body handedness (Kuroda et al. 2009) (Fig. 2). This is a direct proof that the birth of mirror-image animals is not due to the epigenetic consequence but to the chiral positioning of blastomeres at the eight-cell stage. In other words, the twisting of the blastomeres is the very function of the handedness determining gene, and we played the function by our hands though in opposite directions to observe the results of manipulation ("Mechanogenetics").

\section{Nodal pathway common to vertebrates operates}

\section{Continued l-r asymmetric expression of nodal-Pitx genes in chiromorphogenesis}

Asymmetric activation of the Nodal pathway is a conserved feature of deuterostomes for the determination of the asymmetric body plan (Duboc \& Lepage, 2008). In vertebrate, asymmetric expression of nodal occurs in the lateral plate mesoderm transiently at the late developmental stage. The Nodal pathway was found in gastropods as well first in the sinistral snail Biomphalaria glabrata and the dextral snail Lottia gigantea (Grande \& Patel, 2009). We cloned the orthologues of nodal and its downstream Pitx genes and investigated their expression patterns in L. stagnalis (Abe \& Kuroda, 2013; Kuroda et al. 2009) and P. acuta (Abe et al. 2014) by whole mount in situ hybridization. In 


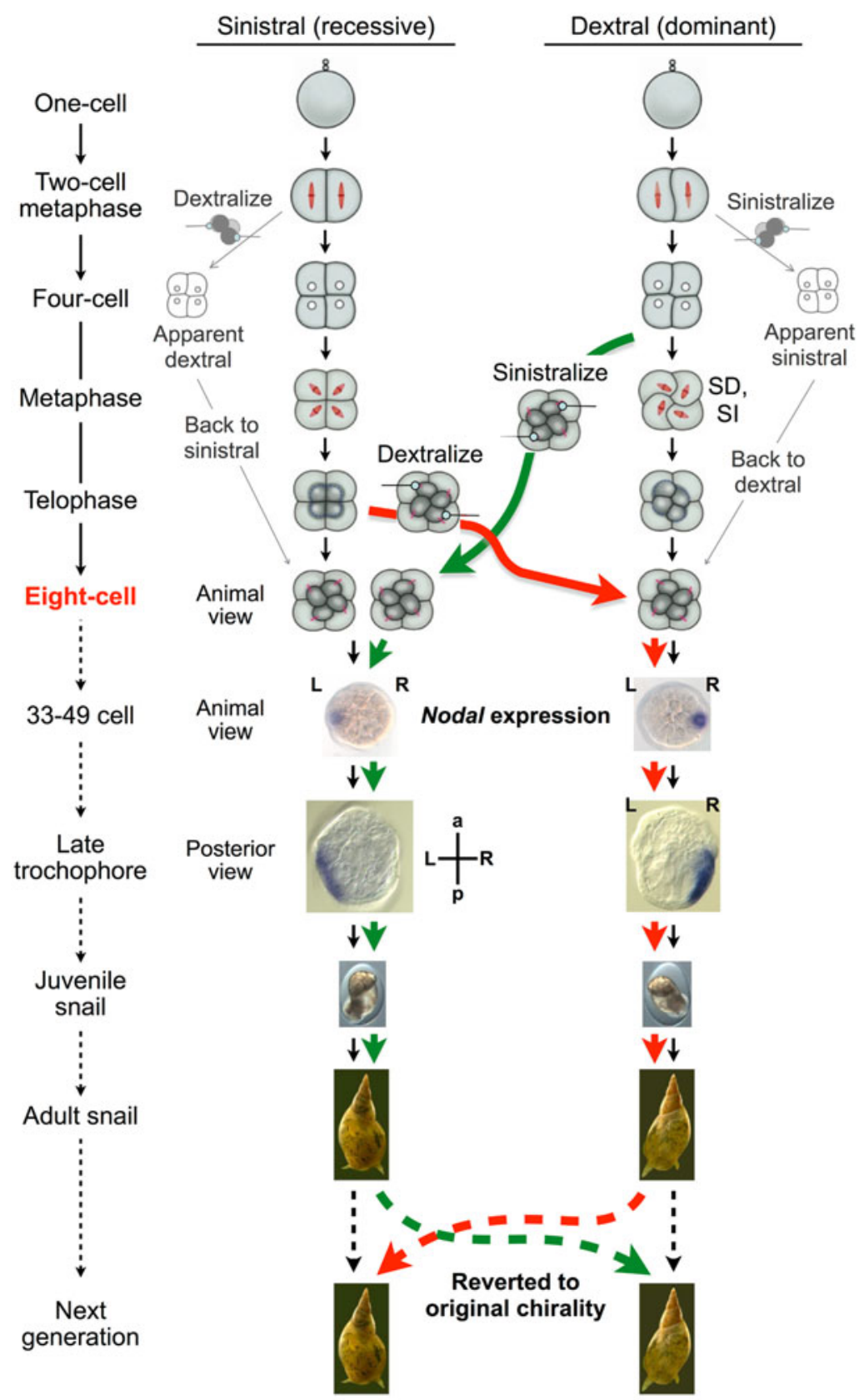

Fig. 2. Chirality-reversed embryos by manipulation at the third cleavage developed to snails with an oppositely-coiled shell and visceral situs inversus. Nodal expression site was inverted by the micromanipulation, throughout the development from the starting 33-49 cell towards the late veliger stage. The chirality of the progenies was dictated by the mother's genotype.

L. stagnalis, nodal and Pitx gene expressions were detected as early as cell stages 33-49 and 49-64, respectively, in a specific blastomere destined to develop into ectoderm. Nodal expression continued up to veliger stage, and then decreased gradually and eventually disappeared when the asymmetrical morphology was noticeable and the shell started to develop. The expression sites for the dextral and sinistral L. stagnalis embryos were invariably mirror images of each other following the third cleavage chirality (Abe \& Kuroda, 2013; Kuroda et al. 2009).

\section{Twisting of blastomeres results in mirror-image Nodal expression}

Embryos of progenies of sinistral and dextral $\mathrm{F}_{7}$ congenic snails exhibited asymmetric nodal and Pitx patterns exactly the same as wild type, indicating that the Nodal pathway acts downstream of the handedness-determining gene product(s) (Kuroda et al. 2009). Remarkably, when chirality at the eight-cell stage was reversed by micromanipulation, the nodal and Pitx expression patterns were completely reversed 


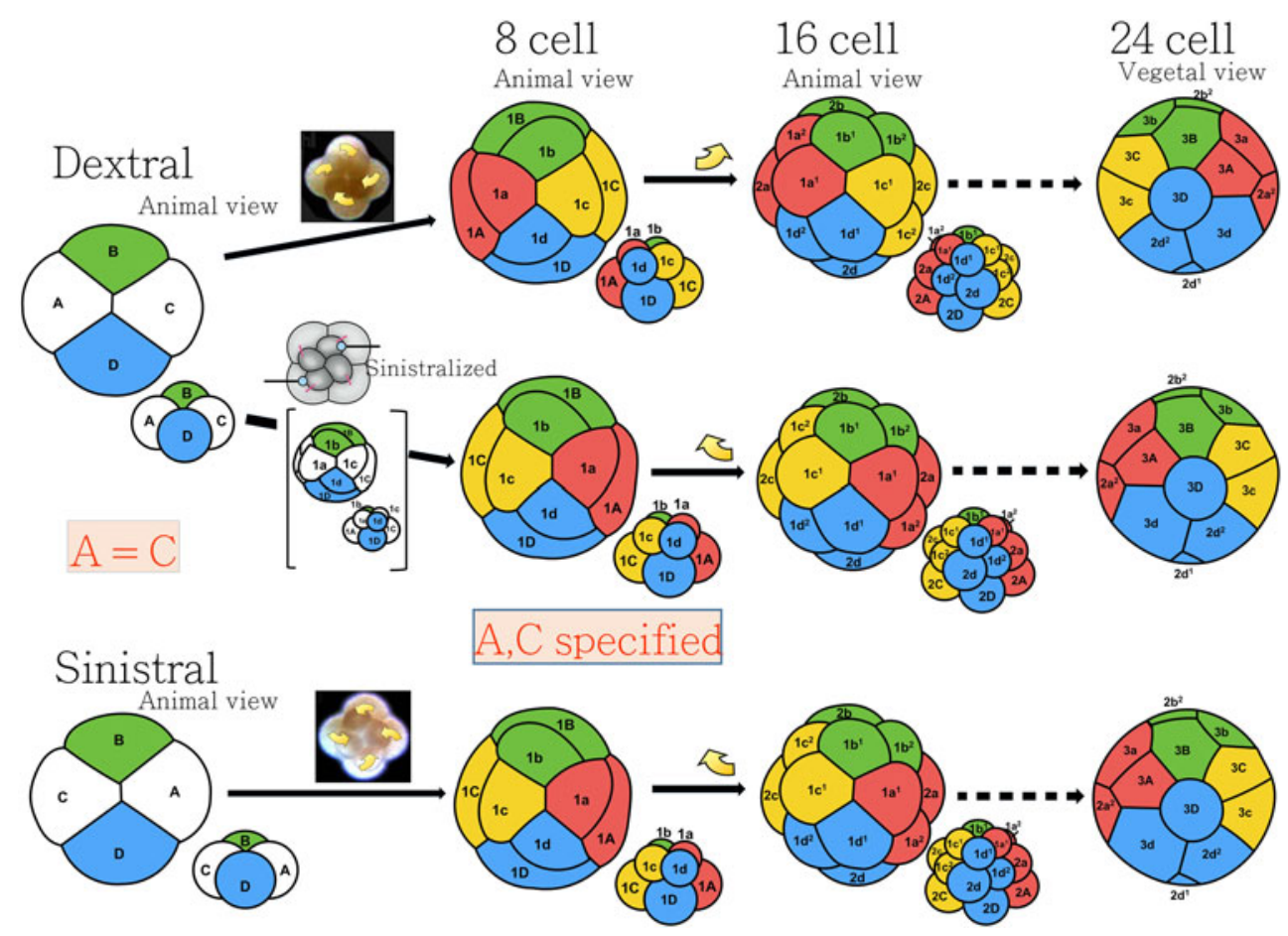

Fig. 3. Blastomere arrangements during the spiral cleavages for the dextral and sinistral embryos and the consequence of micromanipulation at the third cleavage. A-C distinction is established at the eight-cell stage.

(Fig. 2) (Abe \& Kuroda, 2013; Kuroda et al. 2009). Thus, the maternally-determined blastomere arrangement at the eightcell stage dictates the zygotic Nodal signalling pathway.

\section{Spiral cleavage conveys chirality information}

We followed and compared the cytoskeletal dynamics at the fourth cleavage in detail for the mechanically reversed and nonmanipulated embryos of $L$. stagnalis and P. acuta. After the recurrent blastomere compaction during post-mitotic phase in which the morphological chirality of embryos was seemingly lost, macromeres (1Q, Q = A, B, C and D) and then micromeres $(1 \mathrm{q}, \mathrm{q}=\mathrm{a}, \mathrm{b}, \mathrm{c}$ and $\mathrm{d})$ underwent spiral cleavages in the opposite sense to the third cleavage, regardless they were nonmanipulated or manipulated, keeping the features of alternating rotation direction in the successive cleavages (Fig. 3) (Abe et al. 2014; Kuroda et al. 2009). The direction of the fourth cleavage was not influenced by intra-cellular event caused by cell pushing, but was determined by micromere (1q)macromere (1Q) contacts established by the third cleavage (Abe et al. 2014). We believe that spiral cleavage is the way to transfer chirality information from the chirality-determining third cleavage to the later developmental stage.

\section{Cell-fate}

Multicellular organisms are derived from a single fertilized egg. Are cell fates somehow predetermined or do cells and tissues interact with one another to orchestrate developmental processes? Spemann's classical experiments on salamander (Spemann \& Mangold, 1924) have shown the existence of an organizer, now called Spemann organizer, that instructs both neutralization and dorsalization, and that cells can adopt their developmental fate according to their position when instructed by other cells. In the gastropods, the macromere $3 \mathrm{D}$ is known to act as an organizer. How and when is the $\mathrm{D}$ quadrant specification made from which $3 \mathrm{D}$ is derived? There are two types of spiral cleavage patterns, i.e. equal and unequal cell divisions in the first and second cleavages. In the unequal cleaving gastropods such as Ilyanassa, D blastomere is much larger than the other A-C cells at the four-cell stage and is easily recognized. In the case of equal cleaving Lymnaea, however, all four blastomeres are of equal size. Blastomeres $\mathrm{A} / \mathrm{C}$ and $\mathrm{B} / \mathrm{D}$ can be identified as $\mathrm{A}$ and $\mathrm{C}$ are in contact at the animal pole along the crossfurrow, whereas $\mathrm{B}$ and $\mathrm{D}$ are in contact at the vegetal pole. But, A or C, and similarly B or D cannot be distinguished from each other from the appearance (Fig. 3). At the 24-cell stage, the macromere 3D moves to the central location of vegetal side, fills almost entire cleavage cavity where it contacts with micromeres including animal-most 1 st quartet $1 q^{1}$ and $1 \mathrm{q}^{2}(\mathrm{q}=\mathrm{a}, \mathrm{b}, \mathrm{c}, \mathrm{d})$. These cell arrangements take place during the long interval between the fifth and sixth cleavages (Meshcheryakov, 1990). Whether the contacts trigger a cell to become 3D or already specified macromere 3D moves to the position is still a matter of debate. This series of events appears to trigger MAPK activation within the $3 \mathrm{D}$ 


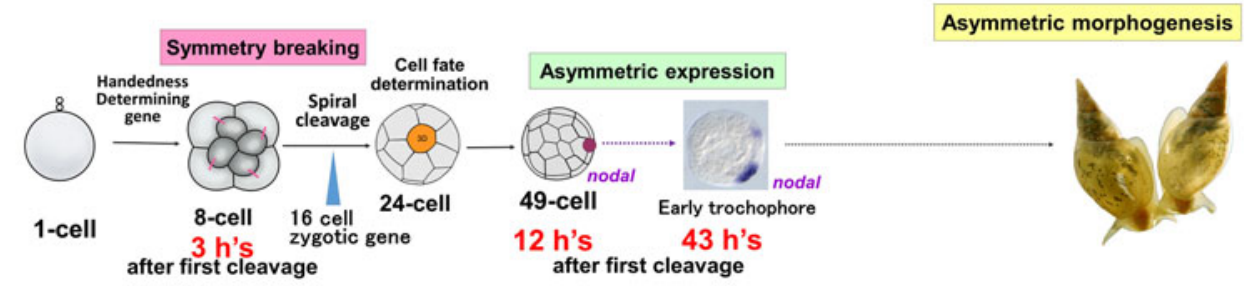

ii) Left-right patterning in vertebrates

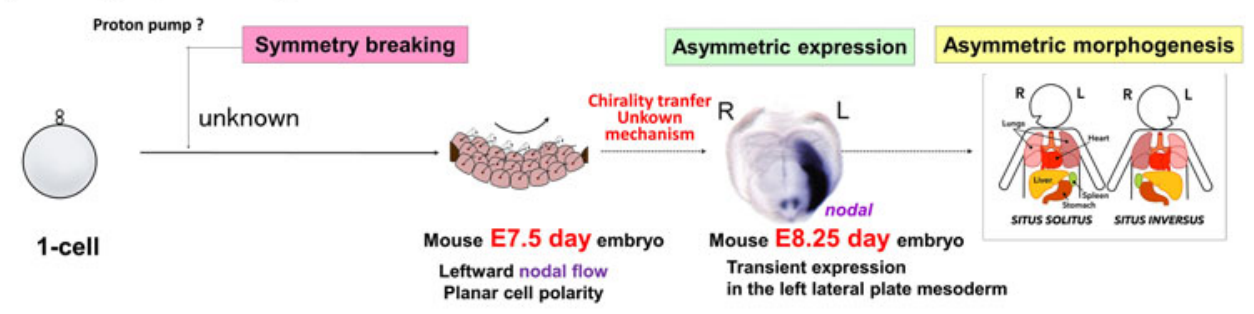

Fig. 4. Snails provide excellent model systems for the study of chiromorphogenesis. Symmetry breaking occurs at the eight-cell stage, and asymmetric expression of nodal gene starts at 33-49 cell, only $12 \mathrm{~h}$ after the first cell cleavage, which can be compared with 8.25 days in the case of mouse.

macromere (Henry \& Perry, 2008), and then MAPK acts upstream of Nodal gene (Lambert \& Nagy, 2003). We are currently analysing the molecular processes of these events.

Blastomere which expresses Nodal gene is conventionally regarded as derived from the macromere C. As described earlier, our micromanipulation at the third cleavage of L. stagnalis shifted the nodal expression to the mirror-image site (Fig. 2), however, this is not because the macromere $\mathrm{C}$ was moved to the other side of midline by twisting (Fig. 3). Our experiments have clearly shown that the cell fate A or C is not yet specified at the four-cell stage. In other words, $1-\mathrm{r}$ symmetry is not yet established before the third cleavage.

\section{Outlook}

The feature of maternal inheritance of chirality, and the correlation of shell coiling and the spiral blastomere cleavage in gastropods were long observed, but, the nature of the link between them had remained obscure. So far, we have clarified (Fig. 4) that the initial step in the 1-r symmetry breaking occurs as early as the third (i.e. the first spiral) cleavage, only $3 \mathrm{~h}$ after the first cleavage of a fertilized egg (before the start of zygotic gene expression), when newly formed micromeres are rotated levotropically and dextrotropically with respected to the macromeres for the sinistral and dextral snails, respectively. Only the dominant chirality shows SD of blastomeres which assures correct micromere-macromere contacts. The single handedness-determining locus dictates the cytoskeletal dynamics at the third cleavage, as proven by our micromanipulation experiments. Chirality thus established is transferred to the cell-fate determination step by spiral cleavages, which end at the 24-cell stage. At this stage, the organizer 3D macromere activates, via still unknown mechanisms, MAPK signal leading to the asymmetric zygotic nodal gene expression at the 33-49 cell stage, only $12 \mathrm{~h}$ after the first cleavage. Nodal pathway is directly involved in asymmetric morphogenesis both in vertebrate and invertebrate. The continued expression of nodal gene in snails is clearly different from the transient expression in vertebrates at the late developmental stage. In the case of mouse, nodal flow and asymmetric nodal expression were observed at E7.5 and E8.25 days, respectively, a long step from the first symmetry breaking (Fig. 4). There are still many questions to be answered. Why and how the spiral cleavages occur in alternating sense? The same mechanisms seem to operate for dominant dextral $L$. stagnalis and sinistral only $P$. acuta. Then, what determines the direction of rotation? What drives levotropic rotation of micromeres in sinistral L. stagnalis after the non-chiral budding of micromeres at the third cleavage? How and when is the organizer 3D macromere's fate determined? How is MAPK activated and how does it lead to Nodal expression?

It is not clear how the asymmetry of animal body plan is associated with homochiral biomolecules. The situation is different from chemical chiromophology where enantiomorphic crystals are made up of enantiomorphic molecules. Lefthand and right-hand shell coiling snails are of course made up of biomolecules of the same unique handedness. Twenty-five years ago, Brown \& Wolpert (1990) proposed a model for handedness development in bilateral animals. Out of their three components, conversion from a handedness at the molecular level to handedness at the cellular and multicellular level (F-molecule) is intriguing, but their model is just an abstract one, devoid of real molecular systems. We have been trying to identify the single handedness-determining gene in 
L. stagnalis by positional cloning. If the gene and its product protein are identified, understanding of chirality determination at the molecular level will be greatly advanced.

\section{References}

Abe, M. \& Kuroda, R. (2013). Continued left-fight asymmetric expression of nodal-Pitx genes throughout the development is required for the gastropod Lymnaea stagnalis's chiromophogenesis, P2-0603. In The 36th Annual Meeting of the Molecular Biology Society of Japan, Kobe, Japan.

Abe, M., Takahashi, H. \& Kuroda, R. (2014). Spiral cleavages determine the left-right body plan by regulating Nodal pathway in monomorphic gastropods, Physa acuta. International Journal of Developmental Biology 58, 513-520. Special issue: "Spiralian Model Systems."

Blum, M., Fiested, K., Thumberger, T. \& Schweickert, A. (2014). The evolution and conservation of left-right patterning mechanisms. Development 141, 1603-1613.

Boycott, A. E., Diver, C. \& Garstang, S. L., Hardy, A. C. \& Turner, F. M. (1930). The inheritance of sinistrality in Lymnaea peregra. Philosophical Transactions of Royal Society of London B 219, 51-131.

Brown, N. A. \& Wolpert, L. (1990). The development of handedness in 1-r asymmetry. Development 109, 1-9.

Coutelis, J-B., Gonzalez-Morales, N., Geminard, C. \& Noselli, S. (2014). Diversity and convergence in the mechanisms establishing $\mathrm{L} / \mathrm{R}$ asymmetry in metazoan. EMBO 15, 926-937.

CRAMPTON, H. E. (1894). Reversal of cleavage in a sinistral gastropod. Annals of the New York Academy of Sciences 8, 167-170.

Duboc, V. \& Lepage, T. (2008). A conserved role for the Nodal signaling pathway in the establishment of dorso-ventral and leftright axes in deuterostomes. Journal of Experimental Zoology (Mol Dev Evol) 310B, 41-53.

Freeman, G. \& Lundelius, J. W. (1982). The developmental genetics of dextrality and sinistrality in the gastropod Lymnaea peregra. Wilhelm Roux Archive Developmental Biology 191, 69-83.

Grande, C. \& Patel, N. H. (2009). Nodal signalling is involved in 1-r asymmetry in snails. Nature 457, 1007-1011.

Henry, J. J. \& Perry, K. J. (2008). MAPK activation and the specification of the D quadrant in the gastropod mollusc, Crepidula fornicata. Developmental Biology 313, 181-195.
Hosotri, Y., Harada, Y. \& Kuroda, R. (2003). Construction of a backcross progeny collection of dextral and sinistral individuals of a freshwater gastropod, Lymnaea stagnalis. Development Genes and Evolution 213, 193-198.

KURODA, R. (2014). How a single gene twists a snail. Integrative and Comparative Biology e54, 677-687.

Kuroda, R., Endo, B., Abe, M. \& Shimizu, M. (2009). Chiral blastomere arrangement dictates zygotic $1-\mathrm{r}$ asymmetry pathway in snails. Nature 462, 790-794.

LAMBERT, J. D \& NAGY, L. M. (2003). The MAPK cascade on equally cleaving spiralian embryos. Developmental Biology 264, 231-241.

Liu, M. M., Davey, J. W., Banerjee, R., Han, J., Yang, F., Aboobaker, A., Blaxter, M. \& Davison, A. (2013). Fine mapping of the pond snail $1-r$ asymmetry (Chirality) locus using RAD-Seq and Fibre-FISH. PLoS ONE 8, e71067.

MeshCHeRYAKov, V. N. (1978). Orientation of the cleavage spindles in pulmonate molluscs. II. The role of the architecture of the intercellular contacts in III and IV cleavage spindle orientation. Ontogenez 9, 567-575.

MeshCHeRYAKov, V. N. (1990). The common pond snail Lymnaea stagnalis. In Animal Species for Developmental Studies, Volume 1. Invertebrates (eds. T. A. Dettlaff \& S. G. Vassetzky), pp. 69-132. New York: Consultants Bureau.

Meshcheryakov, V.N. \& Beloussov, L.V. (1975). Asymmetrical rotations of blastomeres in early cleavage of gastropoda. Wilhelm Roux's Archives 177, 193-203.

Shibazaki, Y., Shimizu, M. \& KURODA, R. (2004). Body handedness is directed by genetically determined cytoskeletal dynamics in the early embryo. Current Biology 14, 1462-1467.

Spemann, H. \& Mangold, H. (1924). Über Induktion von Embryonalanlagen durch Implantation artfremder Organisatoren (Induction of embryonic primordia by implantation of organizers from a different species). Roux's Archiv für Entwicklungsmechanik 100, 599-638.

Sturtevant, A. H. (1923). Inheritance of direction of coiling in Lymnaea. Science 58, 269-270.

VANDENVERG, L. N. \& Levin, M. (2013). A unified model for leftright asymmetry? Comparison and synthesis of molecular models of embryonic laterality. Developmental Biology 379, 1-15.

Verdonk, N. H. \& Van Den Biggelaar, J. A. M. (1983). Early development and the formation of the germ layers. In The Mollusca, Volume 3. Development (eds. N. H. Verdonk, J. A. M. VAN DEN Biggelaar \& A. S. Tompa), pp. 91-122. New York: Academic Press. 